\title{
Global characterization of dose-dependent effects of cadmium in clam Ruditapes philippinarum ${ }^{\text {मै }}$
}

\author{
Junfei Zhan ${ }^{\text {a, d }}$, Shuang Wang ${ }^{\text {a, d }}$, Fei Li ${ }^{\text {a, c }}$, Chenglong Ji ${ }^{\text {a, b, c }}$, Huifeng Wu ${ }^{\text {a, b, c, * }}$ \\ ${ }^{a}$ CAS Key Laboratory of Coastal Environmental Processes and Ecological Remediation, Yantai Institute of Coastal Zone Research (YIC), Chinese Academy of \\ Sciences (CAS); Shandong Key Laboratory of Coastal Environmental Processes, YICCAS, Yantai, 264003, PR China \\ ${ }^{\mathrm{b}}$ Laboratory for Marine Fisheries Science and Food Production Processes, Qingdao National Laboratory for Marine Science and Technology, Qingdao, \\ 266237, PR China \\ ${ }^{\mathrm{c}}$ Center for Ocean Mega-Science, Chinese Academy of Sciences (CAS), Qingdao, 266071, PR China \\ ${ }^{\mathrm{d}}$ University of Chinese Academy of Sciences, Beijing, 100049, PR China
}

\section{A R T I C L E I N F O}

\section{Article history:}

Received 19 July 2020

Received in revised form

20 December 2020

Accepted 28 December 2020

Available online 15 January 2021

\section{Keywords:}

Benchmark dose

Cadmium

Dose-dependent effects

Ruditapes philippinarum

Transcriptomics

\begin{abstract}
A B S T R A C T
Cadmium (Cd) is being frequently detected in marine organisms. However, dose-dependent effects of $\mathrm{Cd}$ challenged unraveling the toxicological mechanisms of $\mathrm{Cd}$ to marine organisms and developing biomarkers. Here, the dose-dependent effects of $\mathrm{Cd}$ on clams Ruditapes philippinarum following exposure to 5 doses of $\mathrm{Cd}(3,9,27,81,243 \mu \mathrm{g} / \mathrm{L})$ were investigated using benchmark dose (BMD) method. By model fitting, calculation of BMD values was performed on transcriptomic profiles, metals concentrations, and antioxidant indices. $\mathrm{Cd}$ exposure induced not only significant $\mathrm{Cd}$ accumulation in clams, but also marked alterations of essential metals such as $\mathrm{Ca}, \mathrm{Cu}, \mathrm{Zn}, \mathrm{Mn}$, and Fe. Gene regulation posed little influence on essential metal homeostasis, indicated by poor enrichment of differentially expressed genes (DEGs) associated with metal binding and metal transport in lower concentrations of Cd-treated groups. BMD analysis on biological processes and pathways showed that peptide cross-linking was the most sensitive biological process to $\mathrm{Cd}$ exposure, followed by focal adhesion, ubiquitin mediated proteolysis, and apoptosis. Occurrence of apoptosis was also confirmed by TUENL-positive staining in gills and hepatopancreas of clams treated with Cd. Furthermore, many DEGs, such as transglutaminases (TGs), metallothionein (MT), STEAP2-like and laccase, which presented linear or monotonic curves and relatively low BMD values, were potentially preferable biomarkers in clams to Cd. Overall, BMD analysis on transcriptomic profiles, metals concentrations and biochemical endpoints unraveled the sensitiveness of key events in response to $\mathrm{Cd}$ treatments, which provided new insights in exploring the toxicological mechanisms of $\mathrm{Cd}$ in clams as well as biomarker selection.
\end{abstract}

() 2021 Elsevier Ltd. All rights reserved.

\section{Introduction}

Due to industrial effluents and river discharge, cadmium (Cd) pollution has been globally observed in marine environment ( $\mathrm{Fu}$ et al., 2014; Naser, 2013). As a non-essential metal, Cd is toxic to aquatic organisms even at low concentrations (Qu et al., 2013; Wang and Rainbow, 2006; Wu et al., 2017). The frequent detection

\footnotetext{
th This paper has been recommended for acceptance by Sarah Harmon.

* Corresponding author. CAS Key Laboratory of Coastal Environmental Processes and Ecological Remediation, Yantai Institute of Coastal Zone Research (YIC), Chinese Academy of Sciences (CAS); Shandong Key Laboratory of Coastal Environmental Processes, YICCAS, Yantai, 264003, PR China.

E-mail address: hfwu@yic.ac.cn (H. Wu).
}

of $\mathrm{Cd}$ in marine organisms is therefore of considerable concern, which creates a pressing need to assess the potential ecological risk. A recent study from our laboratory on metabolomic profiles demonstrated that the altered metabolites presented linear, Ushaped, or inverted U-shaped curves in polychaete Perineresis aibuhitensis treated with a serial of $\mathrm{Cd}$ doses, suggesting the dosedependent effects of Cd (Liu et al., 2019). The dose-dependent effects of Cd challenge the characterization of toxicological mechanisms and developing biomarkers of Cd toxicity.

Dose-response relationship is a necessary part of understanding the cause and effect relationship between chemical exposure levels and biological endpoints. In general, dose-response relationships consist of monotonic responses like linear, power, and exponential curves, as well as non-monotonic responses, such as U-shaped or 
inverted U-shaped curves (Calabrese and Baldwin, 2001). The Ushaped response is characterized by a decrease at low dose and an increase at higher dose, whereas the inverted U-shaped response seems like the opposite of U-shaped curve (Calabrese and Baldwin, 2001). Benchmark dose (BMD) is US EPA's preferred methodology and is becoming the world's standard for dose-response analysis. As an alternative to No-Observed-Adverse-Effect-Level (NOAEL) approach, BMD addresses many limitations of the NOAEL, such as failure to take into consideration the shape of the dose-response curve (Davis et al., 2011). By modeling dose-response relationships and fitting curves, the BMD value that is defined as an endpoint at $x$ percent of the maximal effect can be obtained from dose-response curves and result in a point of departure (POD). Traditional endpoints used for measuring the POD are apical endpoints (e.g., organ weight, lethality or growth rate), which may fail to reflect toxic effects at subtoxic levels. Omics approaches are promising for ecological risk assessment since they enable to provide mechanism information and early warning signals by highthroughput analysis on responses at gene, protein, and metabolite levels (Cappello et al., 2016; Larras et al., 2018). Therefore, doseresponses based on omics data enable to observe early and low concentration-response to chemical exposures and to identify new sensitive biomarkers (Smetanova et al., 2015). Especially, regulated mRNA expression profile following chemical exposure is one of the earliest quantifiable responses, which has been increasingly used to determine the gene BMD value to estimate a chemical's POD (Farmahin et al., 2017). Furthermore, transcriptional gene BMD values could be mapped to biological processes, such as pathways and genes ontology (GO) terms, to allow for the identification of key biological processes that are initiated at specific BMD values (Chauhan et al., 2016).

Marine clams are preferable bioindicators to metal pollution because of its wide distribution, ease of collection, as well as capacity of pollutant accumulation (Cappello et al., 2018; Wang et al., 2012; Zorba et al., 1992). Though marine clams have been frequently utilized for characterization of Cd toxicity and environmental monitoring (Bao et al., 2016; Chora et al., 2009; Ji et al., 2019), poor attention has been paid on the dose-dependent effects of Cd in marine clams. In this work, BMD analysis was conducted on transcriptomic profiles, metals concentrations, and antioxidant indices, aiming to reveal the dose-dependent effects of $\mathrm{Cd}$ in clams. This study will provide new insights in exploring the toxicological mechanisms of $\mathrm{Cd}$ in clams as well as biomarker selection.

\section{Materials and methods}

\subsection{Animals culture and Cd treatments}

Adult clams Ruditapes philippinarum (White pedigree) were purchased from a culturing farm (Qingdao, China). To avoid the effect of size dependence, only those with shell lengths ranging from 3.5 to $3.8 \mathrm{~cm}$ were selected for subsequent exposure experiment. After acclimated in aerated seawater for 5 days, clams were then randomly divided into 6 groups, including control group and 5 Cd-treated groups $\left(3,9,27,81\right.$, and $\left.243 \mu \mathrm{g} / \mathrm{L} \mathrm{Cd}^{2+}\right)$. The clams cultured in the normal filtered seawater were used as control group. The Cd concentrations, containing 3, 9 and $27 \mu \mathrm{g} / \mathrm{L}$, were environmentally relevant (Fu et al., 2014; Zhang et al., 2010), and the highest of $\mathrm{Cd}$ exposure concentration $(243 \mu \mathrm{g} / \mathrm{L})$ referred to about $20 \%$ of $96-\mathrm{h} \mathrm{LC}_{50}(1360 \mu \mathrm{g} / \mathrm{L})$ of $\mathrm{Cd}$ to $R$. philippinarum. The stock solutions of $\mathrm{CdCl}_{2}$ were prepared by dissolving analytical reagent grade $\mathrm{CdCl}_{2}$ in deionized water. Each group consisted of two replicate tanks, each of which was filled with $15 \mathrm{~L}$ seawater and contained 15 clams. All clams used for subsequent analyses were averagely selected from two replicate tanks. During the acclimation and exposure periods, clams were kept in aerated seawater $\left(25 \pm 2{ }^{\circ} \mathrm{C}, 31 \mathrm{ps} \mu\right)$ under a photoperiod of $12 \mathrm{~h}$ light and $12 \mathrm{~h}$ dark, fed with Chlorella vulgari of $2 \%$ tissue dry weight once daily. Seawater was completely replaced by fresh seawater daily. After exposure for 14 days, four clams were selected for histopathological analysis on gill and hepatopancreas, and another 9 clams were selected for measurements of metal contents, antioxidant indices, and transcriptomic analysis. Of the 9 clams, the whole soft tissue of each individual was ground to powder in liquid nitrogen. Each sample was then divided into 3 parts for measurements of metal contents, antioxidant indices, and transcriptomic analysis.

\subsection{Determination of $\mathrm{Cd}$ and essential metals in clam whole body}

The whole soft tissue samples of clams $(n=9)$ were dried at $80{ }^{\circ} \mathrm{C}$ to constant weights and digested in concentrated nitric acid (70\%, Fisher Scientific) heated in water bath overnight. All completely digested samples were diluted with ultrapure water for the determination of $\mathrm{Cd}, \mathrm{Ca}, \mathrm{Mn}, \mathrm{Cu}, \mathrm{Zn}$, and $\mathrm{Fe}$ using ICP-MS technique (Agilent 7500i, Agilent Technologies Co. Ltd, Santa Clara, CA, USA) (Sneddon and Vincent, 2008). Considering that Ca is a macro metal, the measurement of Ca was separately conducted. The limits of quantification (LOQ) of $\mathrm{Cd}, \mathrm{Ca}, \mathrm{Mn}, \mathrm{Cu}, \mathrm{Zn}$, and $\mathrm{Fe}$ in digestion solutions were better than $0.1,10,1,0.1,0.5$, and $5 \mathrm{ppb}$, which were two orders of magnitude lower than those detected in clam samples. The analysis was validated by the standard addition and a reference material. Marine mussel tissue standard reference material (GBW08571) was employed as a certified reference material for metal analysis to ensure internal quality assurance/quality control $(\mathrm{QA} / \mathrm{QC})$ practices. The recovery of target elements, as tested by three individual spiking experiments, was restricted within $90 \%-110 \%$ for all metals mentioned above. Spearman correlation analysis was conducted on $\mathrm{Cd}$ exposure concentrations and other essential metals contents.

\subsection{Transcriptomic analysis}

The sequencing libraries were constructed using commercial assay kit named NEB Next ${ }^{\circledR}$ UltraTM RNA Library Prep Kit for Illumina ${ }^{\circledR}$ (New England Biolabs, MA, USA). A total of 18 sequencing libraries ( 6 groups each containing 3 replicates, each replicate including 3 samples) were constructed following manufacturer's instructions. Due to the limited information of clam genes in National Center for Biotechnology Information (NCBI), the annotation of gene function was carried out based on the following databases in addition to NCBI non-redundant protein sequences and nonredundant nucleotide sequences (Nt): Protein family (Pfam); Clusters of Orthologous Groups of proteins (KOG/COG); Swiss-Prot; Kyoto Encyclopedia of Genes and Genomes (KEGG) Ortholog database (KO); Gene Ontology (GO).

Only genes with $\mid \log _{2}$ fold change $>1$ and Benjamini and Hochberg adjusted $p$ value $<0.05$ were regarded as differentially expressed genes (DEGs). Validation of the transcriptomic analysis was performed with quantitative real-time PCR (qRT-PCR) (Lu et al., 2020). The primers sequences of 6 targeted genes and housekeep gene were listed in Table S1. GO terms and KEGG pathways with adjusted $p$ values less than 0.05 together with at least 3 genes included were considered significantly enriched by DEGs. The details on transcriptomics analysis were available in Supporting Information.

\subsection{Measurement of antioxidant indices}

The antioxidant indices, including glutathione (GSH) and 
glutathione S-transferases (GST, EC 2.5.1.18) of $R$. philippinarum $(n=9)$ were assayed using a multicode microplate reader (infinite M200, TECAN, Switzerland) according to the manufacturer's protocols for enzyme kits (Nanjing Jiancheng Bioengineering Institute, Nanjing, China). The measurement of GSH was based on the increase in absorbance at $412 \mathrm{~nm}$ when 5,5'-dithiobis (2-nitrobenzoic acid) (DTNB) is reduced by GSH (Simth et al., 1988). The GST activity is determined by measuring the consumption of GSH in $1 \mathrm{~min}$. Protein content was measured by BCA assay with bovine serum albumin as standard (Walker, 1994). The unit of GSH was the content of GSH per gram of total protein ( $\mathrm{mg} / \mathrm{g}$ protein), and the unit of the enzyme was defined as the activity of an enzyme per milligram of total protein ( $\mathrm{U} / \mathrm{mg}$ protein).

\subsection{Apoptosis analysis}

After immediate dissection from the clams, gill and hepatopancreas tissues $(n=4)$ were fixed in the Bouin's fixative solution for $24 \mathrm{~h}$, followed by dehydrated in a progressive series of ethanol and embedded in paraffin. Tissues from control and Cd-treated groups were processed together in batches to remove artefacts. Histological sections ( $6-8 \mu \mathrm{m}$ thickness) were cut from the paraffin embedded tissues.

Terminal dUTP nick-end labeling technique (TUNEL-technique) was performed with one step TUNEL kit (Beyotime Institute of Biotechnology, Haimen, Jiangsu, China) according to our previous work (Ji et al., 2014). Briefly, tissue sections were firstly treated with proteinase $\mathrm{K}$. Then the sections were incubated with TUNEL reaction mixture (enzyme terminal transferase (TdT): fluorescein isothiocyanate (FITC)) and propidium iodide (PI), by which apoptosis nuclei were stained in green and red, respectively. Finally, the sections were observed under a laser scanning confocal microscope (Olympus FV 1000, Tokyo, Japan) using $559 \mathrm{~nm}$ and $488 \mathrm{~nm}$ laser for excitation.

\subsection{BMD modeling}

A BMD is a dose or concentration that produces a predetermined change in the response rate of an adverse effect. This predetermined change in response is called the benchmark response (BMR). In this work, BMR was set as $10 \%$ change in the response rate of an adverse effect relative to the response of control group endpoints, including metal contents, antioxidant indices, and DEGs, above control group. By using Benchmark Dose Modeling Software (BMDS, version 3.1.1) proposed by U.S. Environmental Protection Agency, automated BMD modeling analysis was performed on endpoints including metal contents and antioxidant indices, to fit the best model and calculate BMD values of endpoints. A series of curves, including Linear, Polynomial, Power, Hill, Exponential (M2, M3, M4, M5), were fitted to the datasets. Models were initialized using BMDS 3.1.1 model defaults. The selection of best-fit model was relied on the model recommendation decision logic. In brief, the model in viable bin was first taken into consideration. The only candidate model in the viable model bin was of course deemed as the best-fit model. If the viable bin had more than one model, the lowest Akaike information criterion (AIC) or lowest benchmark dose lower confidence limit (BMDL) was selected. If the range of BMDL values was sufficiently close (less than 3-fold different), the AIC value was used; otherwise, the BMDL value was used. If no viable model was recommended, model in questionable bin was also considered and selected according to the selection criterion of model in viable bin.

BMDExpress (version 2.20) was created for the purpose of doseresponse modeling and BMD estimation for transcriptomic data
(Yang et al., 2007). In this work, the DEGs exhibiting significant changes in at least two doses were selected to fit to a series of different models. A best-fit model was identified based on the lowest AIC value as well as goodness-of-fit $P$ value $>0.1$. The Hill model was considered only when $k$ parameter was more than one third of the lowest dose tested. The next best model with goodnessof-fit $P$ value $>0.05$ was selected if a flagged Hill model was selected as the best-fit model based on the above criteria. If the next best model with $P$ value $>0.05$ was not available, the flagged Hill model was retained and modified to 0.5 of the lowest non-flagged Hill BMD value (Thompson et al., 2016). Other parameters for modeling included: power restricted to $\geq 1$, maximum iterations of 250 , and confidence interval of 0.95 . The final selected model was used to calculate a BMD and BMDL. Those genes would be removed if they showed BMD $>$ highest dose, and BMD $<$ one third of the lowest Cd concentration tested. The BMD values of GO terms or KEGG pathways were calculated based on averaging the enriched DEGs' BMD values.

\section{Results}

\subsection{Cd accumulation and essential trace elements in clams}

After exposure for 14 days, the average values of Cd accumulation in clams from 3, 9, 27, 81, and $243 \mu \mathrm{g} / \mathrm{L}$ Cd groups were 4.58, 9.30, 17.1, 31.4 and $48.6 \mu \mathrm{g} / \mathrm{g}$ Cd (dry weight, dw) (Fig. 1 and Table S2). Significant accumulations of Cd were observed in the Cdtreated groups, with an exception for the lowest concentration $(3 \mu \mathrm{g} / \mathrm{L})$ of Cd-treated group. Cd treatments also altered the contents of $\mathrm{Ca}, \mathrm{Fe}, \mathrm{Cu}, \mathrm{Mn}$, and $\mathrm{Zn}$ in clams. With the increase of $\mathrm{Cd}$ concentration for exposure, $\mathrm{Zn}$ and $\mathrm{Cu}$ contents decreased and then stayed consistent, whereas Fe content increased and then kept unchanged. Ca and Mn contents presented U-shaped and inverted U-shaped responses, respectively. Fig. 2 showed a representative response curve of Fe that was fitted to Exp 4 model. By fitting models, BMD calculation demonstrated that $\mathrm{Cd}$ presented the lowest BMD value (0.41) among these metals, followed by $\mathrm{Cu}$ (3.00), $\mathrm{Zn}$ (3.34) and Fe (3.82). The changes of $\mathrm{Ca}$ and $\mathrm{Mn}$ failed to be fitted to a suitable model, and therefore no BMD value could be obtained (Table S2).

Spearman correlation analysis (Table S3) revealed that $\mathrm{Cd}$ $(\mathrm{r}=0.986, P<0.01)$ and $\mathrm{Fe}(\mathrm{r}=0.662, P<0.01)$ contents were positively correlated with $\mathrm{Cd}$ exposure concentrations, whereas $\mathrm{Zn}$ $(\mathrm{r}=-0.632, P<0.01)$ and $\mathrm{Cu}(\mathrm{r}=-0.427, P<0.05)$ contents were negatively correlated with $\mathrm{Cd}$ exposure concentrations. Furthermore, a significantly negative correlation was found between $\mathrm{Ca}$ and $\mathrm{Mn}$ contents $(\mathrm{r}=-0.406, P<0.05)$.

\subsection{Differentially expressed genes in response to Cd treatments}

Compared with control group, a total of 32,340 genes were identified to be differentially expressed with significances (adjusted $p<0.05$ ) in Cd-treated groups. As shown in Fig. 3A, the number of DEGs approximately increased with the exposure concentrations of Cd, and the highest concentration of Cd $(243 \mu \mathrm{g} / \mathrm{L})$ induced most of the DEGs (97.7\%). Furthermore, Venn diagram (Fig. 3B) showed that numerous DEGs were commonly changed in more than one group. qRT-PCR analysis was performed on 6 typical DEGs (transglutaminase 1, metallothionein, receptor of activated kinase $C 1$, serine protease $C$, sarcoplasmic calcium-binding protein, mannan-binding lectin serine protease 1 ) which were significantly altered in at least 3 groups to validate the results of RNA sequencing (Fig. S1). Correlation analysis demonstrated a strong correlation $(\mathrm{r}=0.918, P<0.0001)$ between the data of RNA sequencing and qRT-PCR (Fig. 3C). 

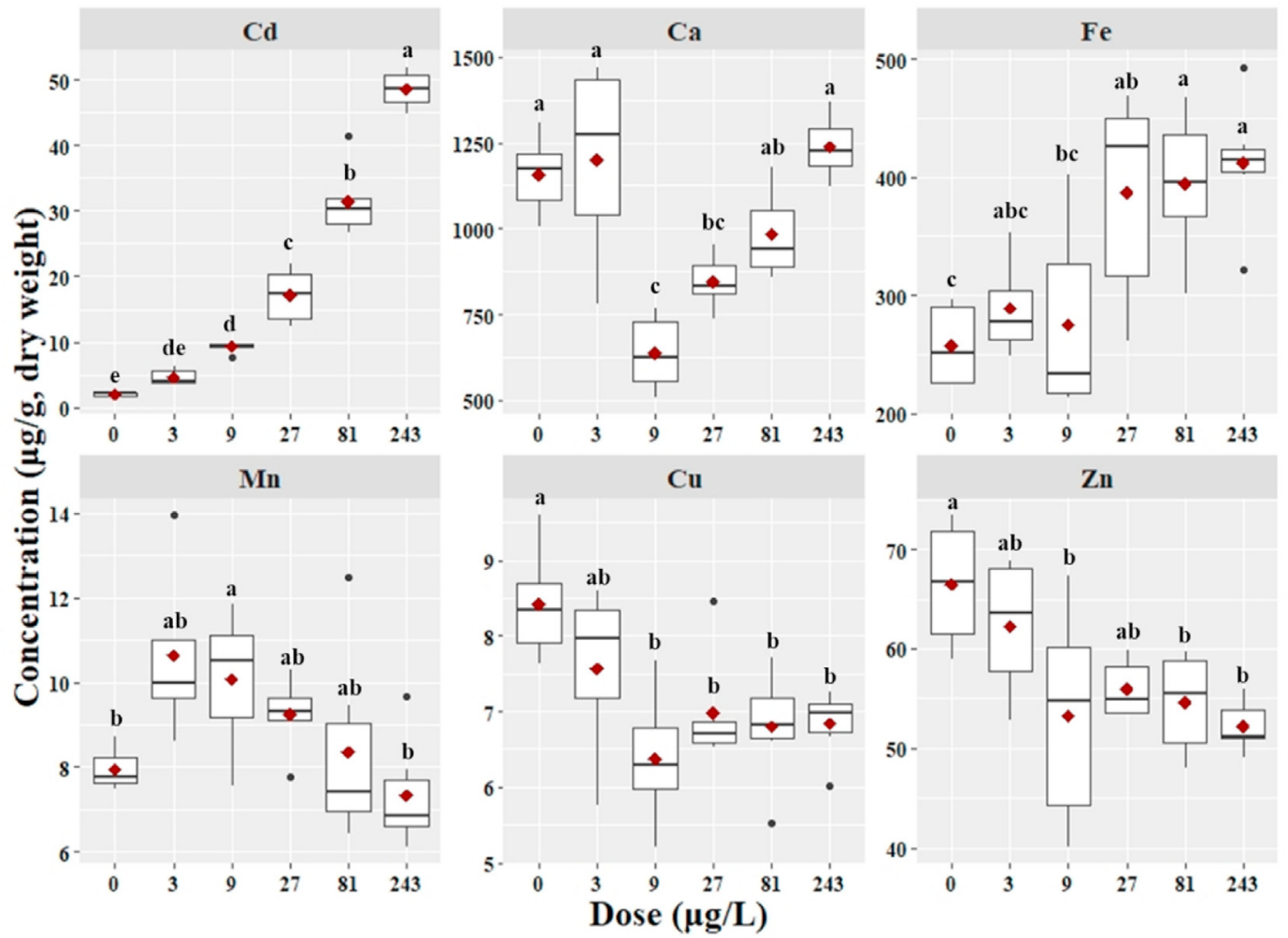

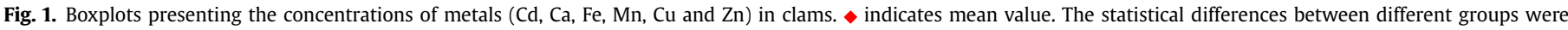
analyzed by one-way analysis of variance (ANOVA) with Tukey post hoc test. Different lowercase letters indicate significant difference between groups.

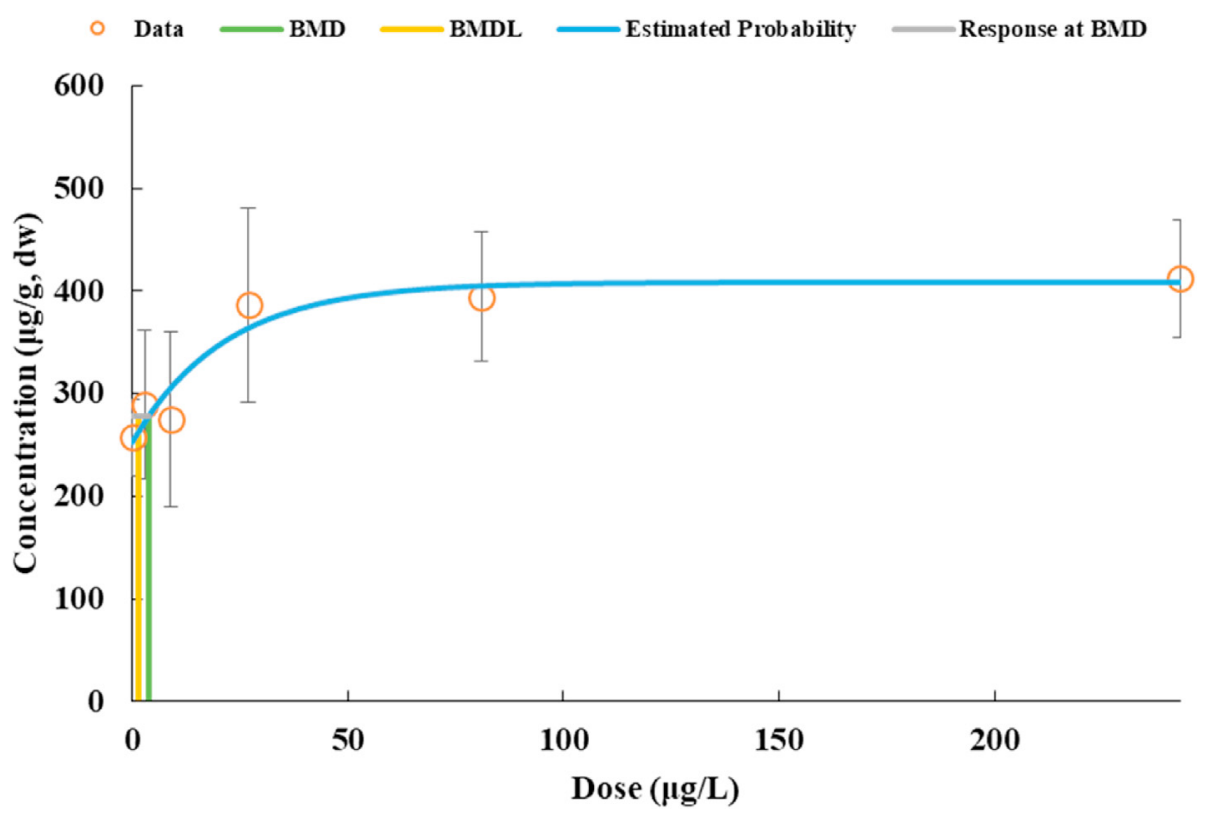

Fig. 2. A representative curve of Fe contents that was best fitted to Exp 4 model.

Model fitting was conducted on 893 genes that were significantly altered in at least two Cd-treated groups. According to the selection criteria mentioned above, a total of 474 genes were fitted to a best model and BMD values were calculated accordingly. The Venn diagram (Fig. 3D) showed the proportion of best-fit models, of which Exp2 (27\%), Poly3 (21\%) and Linear (18\%) were the top 3 bestfit models.

\subsection{GO and KEGG pathway enrichment analyses}

Gene function annotation analysis demonstrated that 32,340 DEGs were involved in 2069 GO terms, including BP, MF, and CC categories, and 230 KEGG pathways, of which $261 \mathrm{GO}$ terms and 53 KEGG pathways were significantly $(P<0.05)$ enriched (Supporting Table). In details, no GO term was significantly $(P<0.05)$ enriched 
A

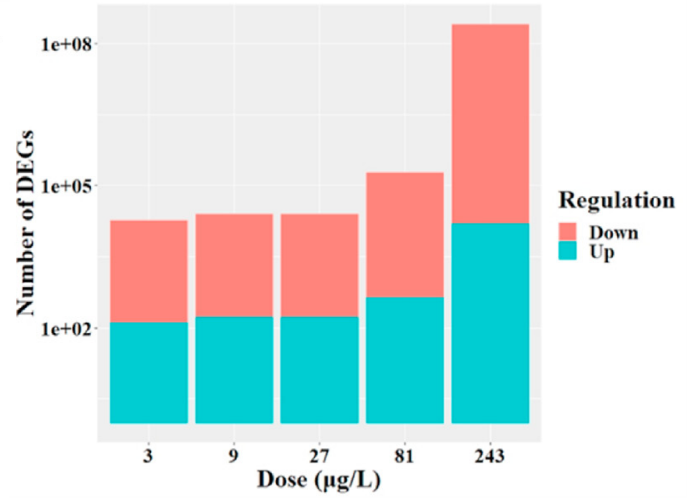

C

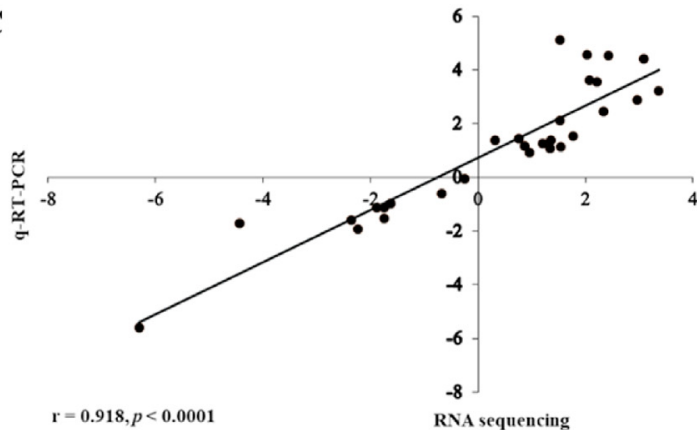

B

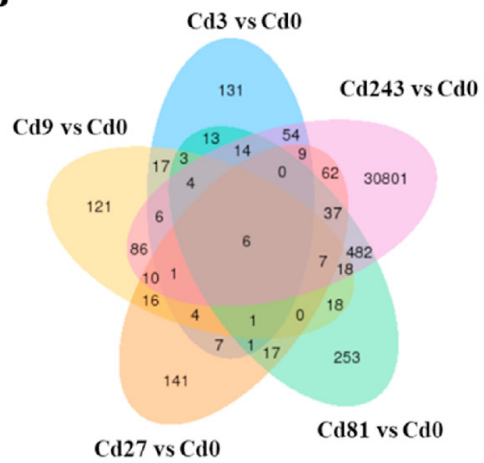

D

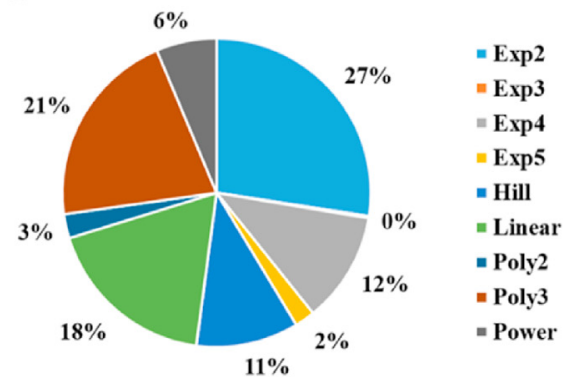

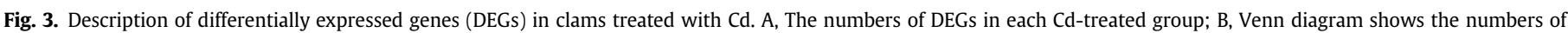

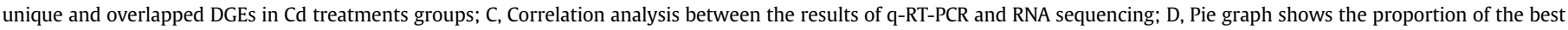
fitted models based on 893 DEGs.

in 3 and $9 \mu \mathrm{g} / \mathrm{L}$ Cd-treated groups, while down-regulation of peptide cross-linking was commonly enriched in 27,81 , and $243 \mu \mathrm{g} / \mathrm{L}$ Cd-treated groups. Like GO terms, no KEGG pathways was significantly enriched in low concentrations of Cd-treated groups (3, 9, and $27 \mu \mathrm{g} / \mathrm{L}$ ). In 81 and $243 \mu \mathrm{g} / \mathrm{L}$ Cd-treated groups, up-regulations of ubiquitin mediated proteolysis, apoptosis and multiple signaling pathways were commonly enriched.

Enrichment analysis was also performed on the DEGs presenting a best-fit model to identify the dose-dependent responses of biological processes and pathways. Significant enrichment (adjusted $P<0.05$ ) was only observed in DEGs which were fitted to Exp2 and Power models (Table 1). Focal adhesion fitted to Exp2 model presented the lowest BMD value (57.3) among the enriched pathways. Apoptosis was commonly fitted to Exp 2 and Power models, and presented BMD values of 92.6 and 141, respectively. Interestingly, ubiquitin mediated proteolysis showed the same fitted models and BMD values. With respect to GO terms, no biological processes were significantly (adjusted $P<0.05$ ) enriched to any model. Only peptide cross-linking with the lowest adjusted $P$ value (0.099) exhibited a BMD value of 17.4.

\subsection{The alterations of antioxidant indices}

The details of antioxidant indices, including glutathione (GSH) and glutathione S-transferase, in control and $\mathrm{Cd}$-treated groups were shown in Fig. 4 and Table S2. The changes of GSH contents in clams showed a U-shaped curve with the increase of Cd exposure concentrations. The highest content of GSH was identified in clams treated with $243 \mu \mathrm{g} / \mathrm{L}$ Cd. Differing from GSH, the activities of GST decreased with the increase of $\mathrm{Cd}$ exposure concentration. However, no significant difference was found in GST activities among control and Cd-treated groups. Furthermore, neither changes of GSH nor GST failed to be fitted to an appropriate model under the selection criteria for best-fit model in this work.

\subsection{Apoptosis in clam gills and hepatopancreas}

As shown in Fig. 5, TUNEL-positive cells that were labeled in green were only observed in gills from $81 \mu \mathrm{g} / \mathrm{L} \mathrm{Cd}$ treatment group (Fig. 5e). In details, the TUNEL-positive cells primarily distributed in the abfrontal zone of gill filaments of $81 \mu \mathrm{g} / \mathrm{L}$ Cd-treated clams. In $243 \mu \mathrm{g} / \mathrm{L}$ Cd-treated group, clam gills exhibited no obvious TUNEL-

Table 1

Enrichment analysis on the DEGs that were fitted to models.

\begin{tabular}{|c|c|c|c|c|c|c|c|c|c|}
\hline Model & Term & Database & ID & Input number & Background number & $P$-Value & Corrected $P$-Value & BMD & BMDL \\
\hline Power & Apoptosis-multiple species & KEGG PATHWAY & ko04215 & 3 & 256 & $4.06 \mathrm{E}-04$ & $4.47 \mathrm{E}-03$ & 141 & 69.3 \\
\hline Power & Ubiquitin mediated proteolysis & KEGG PATHWAY & ko04120 & 3 & 473 & $2.34 \mathrm{E}-03$ & $6.43 \mathrm{E}-03$ & 141 & 69.3 \\
\hline Exp2 & Focal adhesion & KEGG PATHWAY & ko04510 & 7 & 889 & $6.68 \mathrm{E}-04$ & 8.07E-03 & 57.3 & 41.3 \\
\hline Exp2 & Apoptosis-multiple species & KEGG PATHWAY & ko04215 & 4 & 256 & 8.96E-04 & 8.07E-03 & 92.6 & 75.8 \\
\hline Exp2 & NF-kappa B signaling pathway & KEGG PATHWAY & ko04064 & 3 & 235 & 7.09E-03 & $3.54 \mathrm{E}-03$ & 96.6 & 79.0 \\
\hline Exp2 & Ubiquitin mediated proteolysis & KEGG PATHWAY & ko04120 & 4 & 473 & 7.87E-03 & $3.54 \mathrm{E}-03$ & 92.6 & 75.8 \\
\hline Exp2 & peptide cross-linking & GO-biological process & GO:0018,149 & 3 & 70 & $1.96 \mathrm{E}-05$ & $9.95 \mathrm{E}-02$ & 17.4 & 10.0 \\
\hline
\end{tabular}



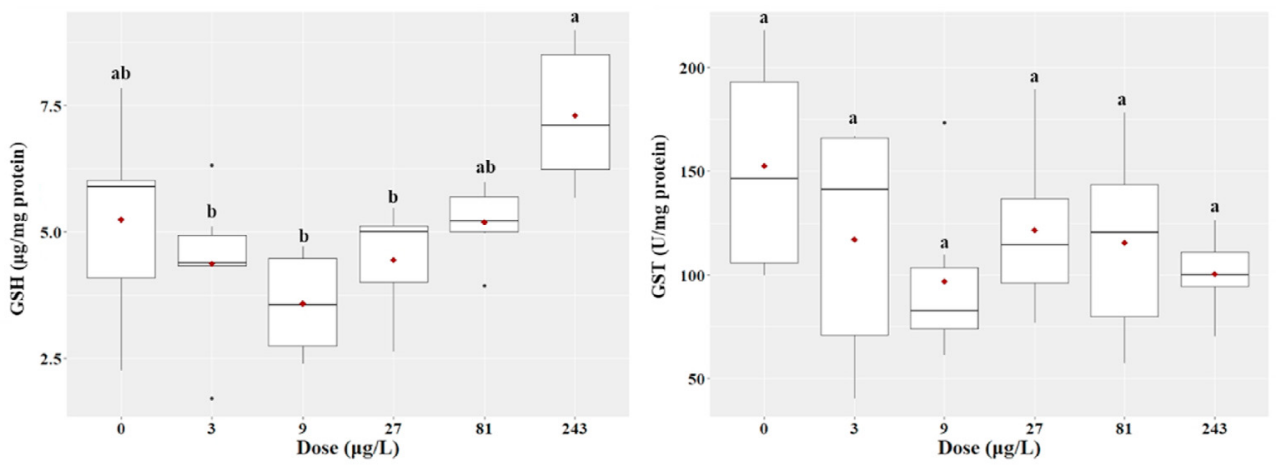

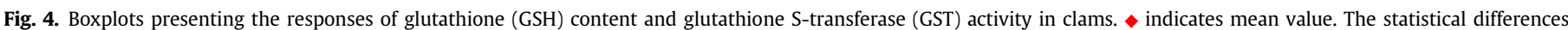

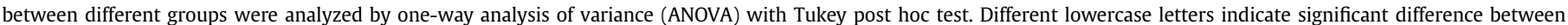
groups.
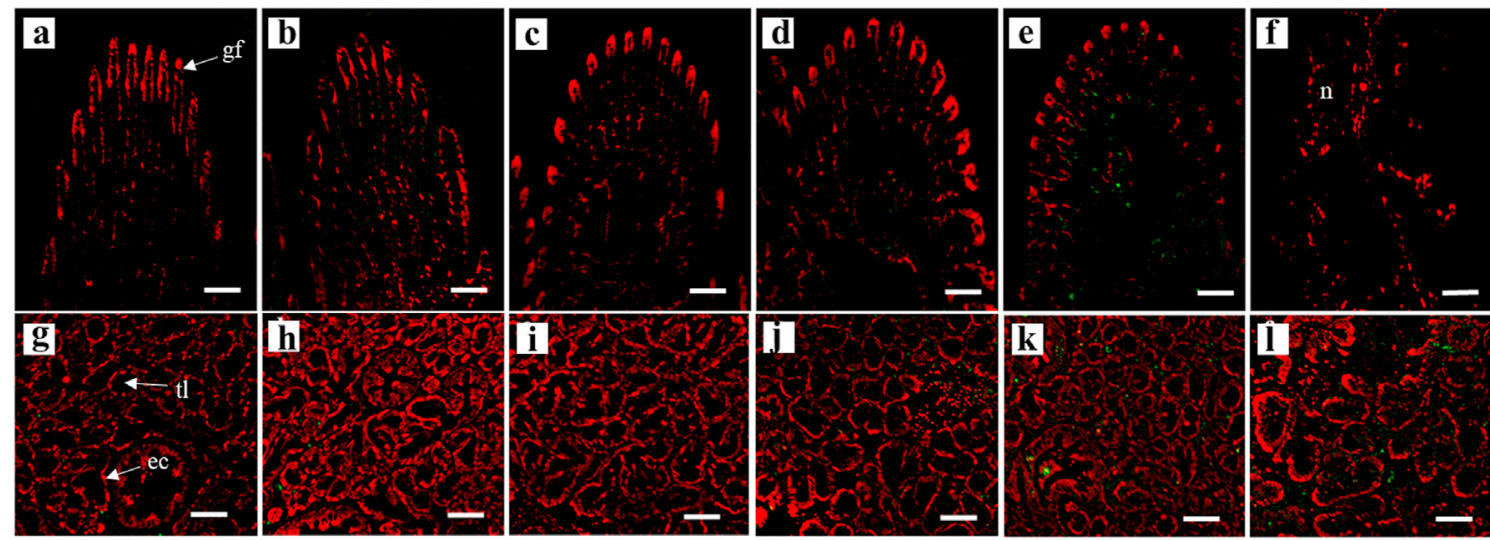

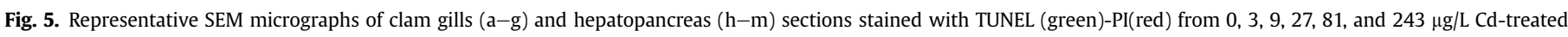

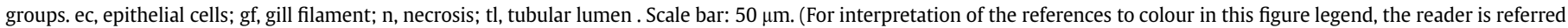
to the Web version of this article.)

positive cells, whereas irregular shape of gill filaments and necrosis of epithelial cells occurred in clams from this treatment (Fig. 5f).

Marked TUNEL-positive cells were also found in clam hepatopancreas from 27, 81, and $243 \mu \mathrm{g} / \mathrm{L} \mathrm{Cd-treated} \mathrm{groups} \mathrm{(Fig.} 5 \mathrm{j}-1$ ). It is noteworthy that most of these cells labeled in green were distributed in intercellular substances. Some TUNEL-positive cells were also observed inside digestive tubules from 81 to $243 \mu \mathrm{g} / \mathrm{L} \mathrm{Cd}-$ treated groups (Fig. 5k-l).

\section{Discussion}

\subsection{The causes and effects of ion homeostasis induced by $\mathrm{Cd}$}

The influences of $\mathrm{Cd}$ on metal homeostasis have been found in cells (Sampels et al., 2017), fish (De Smet et al., 2001) and bacteria (Begg et al., 2015). However, the crucial issue that how Cd dysregulates ion homeostasis has not been fully understood. According to previous studies, Cd influenced ion homeostasis mainly by means of competing for metal transporters and intracellular binding protein as well as gene regulation of ion efflux or/and influx. In general, $\mathrm{Cd}$ accumulation occurs via divalent metal transporters, such as $\mathrm{Ca}^{2+}$ (Hinkle et al., 1987; Tan and Wang, 2011), $\mathrm{Fe}^{2+}$ (Min et al., 2008; Park et al., 2002), and $\mathrm{Mn}^{2+}$ (Begg et al., 2015). In this work, however, Fe contents were unexpectedly elevated with the increase of $\mathrm{Cd}$ exposure concentration. Similar induction of Fe was also found in HepG2 cells treated with a set of $\mathrm{Cd}$ concentrations, with the underlying mechanism that $\mathrm{Cd}$ increased the expression of Fe uptake pathway (Sampels et al., 2017). In this work, genes associated with Fe transport were only altered in $243 \mu \mathrm{g} / \mathrm{L}$ Cd-treated group, suggesting that the elevation of Fe contents was not resulted from the regulation of Fe uptake pathway.

The U-shaped response of $\mathrm{Ca}$ and $\mathrm{Mn}$ contents indicated a hormesis effect of $\mathrm{Cd}$ on $\mathrm{Ca}$ and $\mathrm{Mn}$ homeostasis in clams. The uptake of Ca relies on Ca channel, which was also regarded as the major channel of Cd influx (Craig et al., 1999; Qiu et al., 2005). The decrease of Ca might result from the $\mathrm{Cd}-\mathrm{Ca}$ competition for $\mathrm{Ca}$ channel when the ambient $\mathrm{Cd}$ concentration was relatively low. Other pathways of Ca influx might be activated with the increase of $\mathrm{Cd}$ exposure concentration, leading to the increase of Ca content. After Cd entering cells, intracellular Cd might compete with essential metals like $\mathrm{Cu}$ and $\mathrm{Zn}$ by binding metal buffers like metallothionein (MT), subsequently dysregulated ion homeostasis. MT represents a unique biological principle for buffering the most competitive essential metal ion like $\mathrm{Zn}^{2+}$ and $\mathrm{Cu}^{+}$(Calvo et al., 2017; Krezel and Maret, 2017). Especially, $\mathrm{Zn}$ is the predominant metal bound to MT. Cd has a higher affinity to MT than Zn and can compete to bind to MT in cytoplasm (Romero-Isart and Vasak, 2002). This is one of the detoxification mechanisms of MT. In this work, the decreases of $\mathrm{Cu}$ and $\mathrm{Zn}$ were related to the increased demand of metal buffers for $\mathrm{Cd}$ detoxification.

Ion homeostasis also could be controlled by the expression of pathways for metal uptake, storage and efflux (Begg et al., 2015; Chandrangsu et al., 2017). For example, Cd accumulations in 
Streptococcus pneumoniae led to efflux of $\mathrm{Zn}$ via up-regulation of $\mathrm{Zn}^{2+}$-efflux pathway (Begg et al., 2015). In this work, Ca, Mn, $\mathrm{Cu}$, and $\mathrm{Zn}$ contents were significantly changed when $\mathrm{Cd}$ exposure concentration was as low as $9 \mu \mathrm{g} / \mathrm{L}$, whereas the biological processes associated with metal homeostasis and metal transport were only enriched in the highest concentration $(243 \mu \mathrm{g} / \mathrm{L})$ of $\mathrm{Cd}-$ exposed group, suggesting that the limited effect of gene regulation on these metal turnover in clams. The regulation of ion homeostasis is quite complex, and might be controlled by more than one means (Begg et al., 2015). Hence, the underlying mechanism on dysregulation of ion homeostasis caused by $\mathrm{Cd}$ is still to be determined.

Essential metals play vital roles in numerous physiological processes by maintaining proper protein structure and function and serving as catalytic cofactors for enzymes and as mediators in signaling cascades (Hashimoto and Kambe, 2015). Therefore, the alterations of these essential metals, such as $\mathrm{Ca}, \mathrm{Mn}, \mathrm{Cu}, \mathrm{Zn}$, and $\mathrm{Fe}$ in this work, might subsequently induce the dysregulation of related genes, and even further disrupt intracellular homeostasis and cause toxic effects. For example, iron accumulation increased with the increase of $\mathrm{Cd}$ accumulation, and significant accumulation of iron was observed in $27 \mu \mathrm{g} / \mathrm{L}$ Cd-treated group. Consistently, the gene with a PFAM description of $\mathrm{Fe}^{2+}$ trafficking was up-regulated in 81 and $243 \mu \mathrm{g} / \mathrm{L} \mathrm{Cd}^{2+}$-treated groups. Two genes functioning as iron-sulfur cluster binding were significantly down-regulated when the concentrations of $\mathrm{Cd}$ exceeded $27 \mu \mathrm{g} / \mathrm{L}$. Iron-sulfur cluster has been reported as the target of $\mathrm{Cd}$, and low dose of $\mathrm{Cd}$ could inactivated the iron-sulfur dehydratase family (Xu and Imlay, 2012). Laccase is one of copper-dependent oxidases catalyzing the fourelectron reduction of oxygen to water. In this work, suppression of laccase (laccase-4 and laccase-1-like) expressions begun at the $\mathrm{Cd}$ concentration of $27 \mu \mathrm{g} / \mathrm{L}$, which was positively consistent with the decrease of $\mathrm{Cu}$ contents in clams. Transglutaminases (TGs) are a family of $\mathrm{Ca}^{2+}$-dependent acyl transferases that catalyze crosslinking of proteins during post-translation. TG2 was reported as a $\mathrm{Ca}^{2+}$ sensor and plays a general role in regulation of mitochondrial $\mathrm{Ca}^{2+}$ homeostasis. It can induce the increase of apoptosis in response to $\mathrm{Ca}^{2+}$ overload (Cho et al., 2010). In this work, several TGs were significantly down-regulated in 27,81 , and $243 \mu \mathrm{g} / \mathrm{L} \mathrm{Cd}-$ exposed groups, which might disrupt peptide cross-linking and affect construction of protein.

\subsection{Oxidative stress induced by $\mathrm{Cd}$}

Oxidative stress is a disturbance of the cellular redox balance in favor of the pro-oxidants, and can subsequently lead to disruption of cellular macromolecules (Cuypers et al., 2010). Previous studies have frequently revealed that $\mathrm{Cd}$ exposure induced oxidative stress, despite the inability of $\mathrm{Cd}^{2+}$ to directly produce ROS (Cuypers et al., 2010). The replacement of redox-active elements, such as $\mathrm{Fe}^{2+}$ and $\mathrm{Cu}^{+}$, enables $\mathrm{Cd}$ to indirectly cause oxidative stress (Cuypers et al., 2010). Though the measurements of $\mathrm{Fe}^{2+}$ and $\mathrm{Cu}^{+}$contents were not conducted in this work, its related genes were found to be altered. Coded by the STEAP2, metalloreductase STEAP2 has both ferrireductase and cupric reductase activities. In this work, STEAP2like started to be elevated from 27 to $243 \mu \mathrm{g} / \mathrm{L} \mathrm{Cd}$ treatments and presented a monotonic tendency, suggesting the increased oxidative stress induced by $\mathrm{Cd}$ treatments. Furthermore, $\mathrm{Mn}, \mathrm{Cu}$ and $\mathrm{Zn}$ that function as cofactors are closely associated with superoxide dismutase (SOD) (Zelko et al., 2002). Begg et al. (2015) found that cadmium uptake reduced the millimolar cellular accumulation manganese and zinc in pneumococcus, and thereby increased sensitivity to oxidative stress. In this work, the decreases of $\mathrm{Cu}$ and $\mathrm{Zn}$ in clams might also contribute to oxidative stress.

GSH plays an extremely important role in not only metal detoxification by heavy metal binding and excretion with thiols but also antioxidant defense by ROS scavenging (Jozefczak et al., 2012). Therefore, Cd-induced depletion of the reduced GSH pool contributes to disturbance of the redox balance leading to an oxidative environment (Cuypers et al., 2010). In this work, the alterations of GSH contents presented a gentle U-shaped response. Cd exposure increased the demand of GSH for Cd detoxification via Cd binding. However, when Cd accumulation exceeded the intracellular GSH pool, an increase of GSH was observed in clams induced by higher concentrations of $\mathrm{Cd}$, which probably resulted from activation of GSH production pathway. Moreover, the genes associated with GSH degradation were also altered in response to $\mathrm{Cd}$ exposure. Glutathione-specific gamma-glutamylcyclotransferase 1 (GammaGCG1) is encoded by CHAC1 and responsible for intracellular GSH degradation. A previous study found that overexpression of CHAC1 in yeast led to an $80 \%$ increase in reactive oxygen species (ROS) levels (Kaur et al., 2017). The up-regulation of CHAC1 in clams treated with $\mathrm{Cd}$ indicated the oxidative stress induced by $\mathrm{Cd}$ treatments.

Overall, the decrease of GSH contents and up-regulations of genes MT, STEAP2-like, CHAC1 and GST in clams confirmed the increasing demands of $-\mathrm{SH}$ groups for $\mathrm{Cd}$ detoxification and induction of oxidative stress. Especially, MT, STEAP2-like and CHAC1 presented monotonic up-regulations with the increase of $\mathrm{Cd}$ concentrations, suggesting that these genes were preferable biomarkers in clam to Cd treatment.

\subsection{Cd disrupted signal transduction and induced apoptosis and necrosis}

Focal adhesions anchor the cell to the extracellular matrix and can mediate both mechanical and biochemical signaling, which play a critical role in regulating cell proliferation and apoptosis (Burridge, 2017). In this work, down-regulation of DEGs associated with focal adhesion was found to be enriched in $81 \mu \mathrm{g} / \mathrm{L}$ Cd-treated group. According to the BMD analysis performed on the KEGG pathways, focal adhesion had the lowest of BMD value, suggesting that focal adhesion was the most sensitive pathway in response to $\mathrm{Cd}$ treatments. The disruption of focal adhesion was also found in mesangial cells treated with $\mathrm{Cd}$, which was associated at least in part with $\mathrm{Ca}^{2+}$ /calmodulin-dependent protein kinase II (Choong et al., 2013). $\mathrm{Ca}^{2+}$ plays essential roles in cell signal transduction. In this work, Ca contents and calmodulin expression were significantly altered in clams treated with $\mathrm{Cd}$, which induced dysregulation of cell signaling like focal adhesion, subsequently led to proliferation or apoptosis.

Apoptosis is a fundamental form of programmed cell death, which is involved in development and aging and acts as a homeostatic mechanism to maintain cell populations in multicellular organisms. Heavy metals could induce apoptosis, which always occurred together with over-production of ROS and oxidation effect (Hamada et al., 1997; Morcillo et al., 2016; Xia et al., 2016). In this work, up-regulation of genes associated with apoptosis was significantly enriched in 81 and $243 \mu \mathrm{g} / \mathrm{L}$ Cd-treated groups. BMD analysis revealed that apoptosis related genes could be fitted to more than one model, meaning that apoptosis was a dosedependent biological process. With respect to clams, both gill and hepatopancreas are typical targets for marine contaminants. TUNEL staining conducted on gills and hepatopancreas confirmed the occurrence of apoptosis in clams treated with 81 and $243 \mu \mathrm{g} / \mathrm{L} \mathrm{Cd}$. It is noteworthy that necrosis occurred in gills and hepatopancreas in clams treated with the highest concentration $(243 \mu \mathrm{g} / \mathrm{L})$ of $\mathrm{Cd}$. Necrosis is considered to be unregulated and accidental cell death, which is characterized by the expansion of cellular organelles and plasma membrane rupture. In general, apoptosis and necrosis 
interplay and co-exist in response to xenobiotics. Apoptosis usually occurs after short time of metal exposure, whereas persistent exposure tends to cause necrosis (Huk et al., 2004; Wang et al., 2013).

\subsection{Dose-dependent effects and biomarker selection}

BMD method is increasingly used to assess health risk of environmental contaminants (Filipsson et al., 2003; Wang et al., 2016). In this work, multiple dose-response relationships indicated by various curves of metals and DEGs suggested the complexity of responsive mechanisms in clams to $\mathrm{Cd}$ exposure. Concerning metals, Cd accumulation occurred at low concentrations of $\mathrm{Cd}$ exposure, while changes of essential metals occurred at higher concentrations of $\mathrm{Cd}$ exposure. $\mathrm{Cu}, \mathrm{Zn}$ and $\mathrm{Fe}$ contents showed monotonic tendencies and low BDM values in response to $\mathrm{Cd}$ treatments, indicating that these metals in clams were highly sensitive to $\mathrm{Cd}$ exposure. Though $\mathrm{Ca}$ and $\mathrm{Mn}$ failed to fit the best model under the selection standard of BMD method, the U-shaped or inverted U-shaped responses of these two metals signified a hormesis induced by $\mathrm{Cd}$. With respect to DEGs and its related biological processes and pathways, peptide cross-linking presented the lowest BMD value, followed by focal adhesion, ubiquitin mediated proteolysis, and apoptosis. The order of these responses provided a better understanding of the toxicological mechanisms of Cd. However, due to only focusing on linear, polynomial, hill, exponential and power models, BMD method has the drawback in describing sigmoid or U-shaped responses, which led to failures to fit response curves with many DEGs and obtain BMD values. In this work, only half proportion (474/893) of DEGs were fitted to a best model and obtained the BMD values. Therefore, many DEGs such as STEAP2-like and CHAC1 that failed to fit a best model were also taken into consideration for explanation of toxicological mechanisms of $\mathrm{Cd}$.

Biomarkers have the potential in monitoring and risk assessment. Characterization of dose-response relationship and calculation of BMD values also allow for selection of preferable biomarkers. In this work, many DEGs, such as TGs, MT, STEAP2-like and laccase, which have linear or monotonic curves and relatively low BMD values, were potentially preferable biomarkers in clams to Cd. By contrast, many traditional biomarkers such as GSH content and GST activity in clams seemed not to be suitable for bioindicating Cd because of its non-linear response curves. Certainly, further confirmation in samples from field environment is quite essential.

\section{Conclusion}

This is the first study to investigate the dose-dependent transcriptomic and biochemical responses in clams exposed to $\mathrm{Cd}$ for 14 days through BMD method. Cd exposure induced not only significant Cd accumulation in clams, but also marked alterations of essential metals such as $\mathrm{Ca}, \mathrm{Cu}, \mathrm{Zn}, \mathrm{Mn}$, and Fe. Cd accumulation presented the lowest of BMD value, followed by $\mathrm{Cu}, \mathrm{Zn}$ and Fe. The U-shaped or inverted U-shaped responses of Ca and Mn signified a hormesis induced by $\mathrm{Cd}$. We also found that the alterations of essential metals were not attributed to gene regulation when clams was treated with low concentrations of $\mathrm{Cd}$. Peptide cross-linking was the most sensitive biological process, followed by focal adhesion, ubiquitin mediated proteolysis, and apoptosis. Furthermore, many DEGs, such as TGs, MT, STEAP2-like and laccase, were potentially preferable biomarkers in clams to Cd. Overall, BMD analysis performed on transcriptomic profiles and biochemical endpoints revealed the sensitiveness of key events in response to $\mathrm{Cd}$ exposure and provided new insights in exploring the toxicological mechanisms of Cd in clams as well as biomarker selection.

\section{Author statement}

Junfei Zhan: Formal analysis, Writing - original draft preparation; Shuang Wang: Writing-Reviewing and Editing; Fei Li: Writing-Reviewing and Editing; Chenglong Ji: Funding acquisition, Writing-Reviewing and Editing; Huifeng Wu: Funding acquisition, Writing-Reviewing and Editing, Project administration

\section{Declaration of competing interest}

The authors declare that they have no known competing financial interests or personal relationships that could have appeared to influence the work reported in this paper.

\section{Acknowledgments}

This research was supported by the grants from National Natural Science Foundation of China (42076164 and 41676114) and the Young Taishan Scholars Program of Shandong Province for Prof. Huifeng Wu (tsqn201812115).

\section{Appendix A. Supplementary data}

Supplementary data to this article can be found online at https://doi.org/10.1016/j.envpol.2021.116443.

\section{References}

Bao, Y., Liu, X., Zhang, W., Cao, J., Li, W., Li, C., Lin, Z., 2016. Identification of a regulation network in response to cadmium toxicity using blood clam Tegillarca granosa as model. Sci. Rep. 6, 35704.

Begg, S.L., Eijkelkamp, B.A., Luo, Z., Counago, R.M., Morey, J.R., Maher, M.J. Ong, C.L.Y., McEwan, A.G., Kobe, B., O’Mara, M.L., Paton, J.C., McDevitt, C.A., 2015. Dysregulation of transition metal ion homeostasis is the molecular basis for cadmium toxicity in Streptococcus pneumoniae. Nat. Commun. 6, 6418.

Burridge, K., 2017. Focal adhesions: a personal perspective on a half century of progress. FEBS J. 284, 3355-3361.

Calabrese, E.J., Baldwin, L.A., 2001. U-shaped dose-responses in biology, toxicology, and public health. Annu. Rev. Publ. Health 22, 15-33.

Calvo, J., Jung, H.M., Meloni, G., 2017. Copper metallothioneins. IUBMB Life 69, $236-245$.

Cappello, T., Giannetto, A., Parrino, V., Maisano, M., Oliva, S., Marco, G., Guerriero, G. Mauceri, A., Fasulo, S., 2018. Baseline levels of metabolites in different tissues of mussel Mytilus galloprovincialis (Bivalvia: mytilidae). Comp. Biochem. Physiol. D 26, 32-39.

Cappello, T., Pereira, P., Maisano, M., Mauceri, A., Pacheco, M., Fasulo, S., 2016. Advances in understanding the mechanisms of mercury toxicity in wild golden grey mullet (Lizaaurata) by 1H NMR-based metabolomics. Environ. Pollut. 219, $139-148$

Chandrangsu, P., Rensing, C., Helmann, J.D., 2017. Metal homeostasis and resistance in bacteria. Nat. Rev. Microbiol. 15, 338-350.

Chauhan, V., Kuo, B., McNamee, J.P., Wilkins, R.C., Yauk, C.L., 2016. Transcriptional benchmark dose modeling: exploring how advances in chemical risk assessment may be applied to the radiation field. Environ. Mol. Mutagen. 57, 589-604.

Cho, S.Y., Lee, J.H. Bae, H.D. Jeong E.M. Jang G.Y, Kim, C.W Shin, D.M., Jeon, J.H., Kim, I.G., 2010. Transglutaminase 2 inhibits apoptosis induced by calciumoverload through down-regulation of Bax. Exp. Mol. Med. 42, 639-650.

Choong, G., Liu, Y., Templeton, D.M., 2013. Cadmium affects focal adhesion kinase (FAK) in mesangial cells: involvement of CaMK-II and the actin cytoskeleton. J. Cell. Biochem. 114, 1832-1842.

Chora, S., Starita-Geribaldi, M., Guigonis, J.M., Samson, M., Romeo, M., Bebianno, M.J., 2009. Effect of cadmium in the clam Ruditapes decussatus assessed by proteomic analysis. Aquat. Toxicol. (Amst.) 94, 300-308.

Craig, A., Hare, L., Tessier, A., 1999. Experimental evidence for cadmium uptake via calcium channels in the aquatic insect Chironomus staegeri. Aquat. Toxicol. (Amst.) 44, 255-262.

Cuypers, A., Plusquin, M., Remans, T., Jozefczak, M., Keunen, E., Gielen, H., Opdenakker, K., Nair, A.R., Munters, E., Artois, T.J., Nawrot, T., Vangronsveld, J., Smeets, K., 2010. Cadmium stress: an oxidative challenge. Biometals 23, 927-940.

Davis, J.A., Gift, J.S., Zhao, Q.J., 2011. Introduction to benchmark dose methods and U.S. EPA's benchmark dose software (BMDS) version 2.1.1. Toxicol. Appl. 
Pharmacol. 254, 181-191.

De Smet, H., De Wachter, B., Lobinski, R., Blust, R., 2001. Dynamics of (Cd,Zn)metallothioneins in gills, liver and kidney of common carp Cyprinus carpio during cadmium exposure. Aquat. Toxicol. (Amst.) 52, 269-281.

Farmahin, R., Williams, A., Kuo, B., Chepelev, N.L., Thomas, R.S., BartonMaclaren, T.S., Curran, I.H., Nong A., Wade, M.G., Yauk, C.L., 2017. Recommended approaches in the application of toxicogenomics to derive points of departure for chemical risk assessment. Arch. Toxicol. 91, 2045-2065.

Filipsson, A.F., Sand, S., Nilsson, J., Victorin, K., 2003. The benchmark dose methodReview of available models, and recommendations for application in health risk assessment. Crit. Rev. Toxicol. 33, 505-542.

Fu, J., Wang, H., Billah, S.M.R., Yu, H.X., Zhang, X.W., 2014. Heavy metals in seawater, sediments, and biota from the coastal area of Yancheng city, China. Environ. Toxicol. Chem. 33, 1697-1704.

Hamada, T., Tanimoto, A., Sasaguri, Y., 1997. Apoptosis induced by cadmium. Apoptosis 2, 359-367.

Hashimoto, A., Kambe, T., 2015. Mg, Zn and Cu transport proteins: a brief overview from physiological and molecular perspectives. J. Nutr. Sci. Vitaminol. 61, 116-118.

Hinkle, P.M., Kinsella, P.A., Osterhoudt, K.C., 1987. Cadmium uptake and toxicity via voltage-sensitive calcium channels. J. Biol. Chem. 262, 16333-16337.

Huk, O.L., Catelas, I., Mwale, F., Antoniou, J., Zukor, D.J., Petit, A., 2004. Induction of apoptosis and necrosis by metal ions in vitro. J. Arthroplasty 19, 84-87.

Ji, C., Wu, H., Wei, L., Zhao, J., 2014. iTRAQ-based quantitative proteomic analyses on the gender-specific responses in mussel Mytilus galloprovincialis to tetrabromobisphenol A. Aquat. Toxicol. 157, 30-40.

Ji, C., Lu, Z., Xu, L., Li, F., Cong, M., Shan, X., Wu, H., 2019. Evaluation of mitochondrial toxicity of cadmium in clam Ruditapes philippinarum using iTRAQ-based proteomics. Environ. Pollut. 251, 802-810.

Jozefczak, M., Remans, T., Vangronsveld, J., Cuypers, A., 2012. Glutathione is a key player in metal-induced oxidative stress defenses. Int. J. Mol. Sci. 13, 3145-3175.

Kaur, A., Gautam, R., Srivastava, R., Chandel, A., Kumar, A., Karthikeyan, S., Bachhawat, A.K., 2017. ChaC2, an enzyme for slow turnover of cytosolic glutathione. J. Biol. Chem. 292, 638-651.

Krezel, A., Maret, W., 2017. The functions of metamorphic metallothioneins in zinc and copper metabolism. Int. J. Mol. Sci. 18, 1237.

Liu, F., Lu, Z., Wu, H., Ji, C., 2019. Dose-dependent effects induced by cadmium in polychaete Perinereis aibuhitensis. Ecotoxicol. Environ. Saf. 169, 714-721.

Lu, Z., Huang, W., Wang, S., Shan, X., Ji, C., 2020. Liver transcriptome analysis reveals the molecular responses to low-salinity in large yellow croaker Larimichthys crocea. Aquaculture 517, 734827.

Min, K.S., Iwata, N., Tetsutikawahara, N., Onosaka, S., Tanaka, K., 2008. Effect of hemolytic and iron-deficiency anemia on intestinal absorption and tissue accumulation of cadmium. Toxicol. Lett. 179, 48-52.

Morcillo, P., Esteban, M.A., Cuesta, A., 2016. Heavy metals produce toxicity, oxidative stress and apoptosis in the marine teleost fish SAF-1 cell line. Chemosphere $144,225-233$.

Naser, H.A., 2013. Assessment and management of heavy metal pollution in the marine environment of the Arabian Gulf: a review. Mar. Pollut. Bull. 72, 6-13.

Park, J.D., Cherrington, N.J., Klaassen, C.D., 2002. Intestinal absorption of cadmium is associated with divalent metal transporter 1 in rats. Toxicol. Sci. 68, 288-294.

Qiu, J., Xie, Z., Wang, W.-X., 2005. Effects of calcium on the uptake and elimination of cadmium and zinc in Asiatic clams. Arch. Environ. Contam. Toxicol. 48, $278-287$.

Qu, R., Wang, X., Feng, M., Li, Y., Liu, H., Wang, L., Wang, Z., 2013. The toxicity of cadmium to three aquatic organisms (Photobacterium phosphoreum, Daphnia magna and Carassius auratus) under different pH levels. Ecotoxicol. Environ. Saf.
95, 83-90.

Romero-Isart, N., Vasak, M., 2002. Advances in the structure and chemistry of metallothioneins. J. Inorg. Biochem. 88, 388-396.

Sampels, S., Kroupova, H.K., Linhartova, P., 2017. Effect of cadmium on uptake of iron, zinc and copper and mRNA expression of metallothioneins in HepG2 cells in vitro. Toxicol. Vitro 44, 372-376.

Smetanova, S., Riedl, J., Zitzkat, D., Altenburger, R., Busch, W., 2015. Highthroughput concentration-response analysis for omics datasets. Environ. Toxicol. Chem. 34, 2167-2180.

Sneddon, J., Vincent, M., 2008. ICP-OES and ICP-MS for the determination of metals: application to oysters. Anal. Lett. 41, 1291-1303.

Simth, I.K., Vierheller, T.L., Thorne, C.A., 1988. Assay of glutathione reductase in crude tissue homogenates using 5,5'-dithiobis(2-nitrobenzoic acid). Anal. Biochem. 175, 408-413.

Tan, Q., Wang, W.-X., 2011. Acute toxicity of cadmium in Daphnia magna under different calcium and $\mathrm{pH}$ conditions: importance of influx rate. Environ. Sci. Technol. 45, 1970-1976.

Thompson, C.M., Rager, J.E., Suh, M., Ring, C.L., Proctor, D.M., Haws, L.C., Fry, R.C., Harris, M.A., 2016. Transcriptomic responses in the oral cavity of F344 rats and B6C3F1 mice following exposure to $\mathrm{Cr}(\mathrm{VI})$ : implications for risk assessment. Environ. Mol. Mutagen. 57, 706-716.

Walker, J., 1994. The bicinchoninic acid (BCA) assay for protein quantitation. In: Walker, J.M. (Ed.), Basic Protein and Peptide Protocols. Methods in Molecular Biology ${ }^{\mathrm{TM}}$, vol. 32. Humana Press.

Wang, J., Zhang, P., Shen, Q., Wang, Q., Liu, D., Li, J., Wang, L., 2013. The effects of cadmium exposure on the oxidative state and cell death in the gill of freshwater crab Sinopotamon henanense. PloS One 8, e64020.

Wang, W.-X., Rainbow, P.S., 2006. Subcellular partitioning and the prediction of cadmium toxicity to aquatic organisms. Environ. Chem. 3, 395-399.

Wang, X., Wang, Y., Feng, L., Tong, Y., Chen, Z., Ying, S., Chen, T., Li, T., Xia, H., Jiang, Z. Shang, Q., Lou, X., Lou, J., 2016. Application of the benchmark dose (BMD) method to identify thresholds of cadmium-induced renal effects in nonpolluted areas in China. PloS One 11, e0161240.

Wang, Z., Yan, C., Vulpe, C.D., Yan, Y., Chi, Q., 2012. Incorporation of in situ exposure and biomarkers response in clams Ruditapes philippinarum for assessment of metal pollution in coastal areas from the Maluan Bay of China. Mar. Pollut. Bull. 64, 90-98.

Wu, H., Xu, L., Yu, D., Ji, C., 2017. Differential metabolic responses in three life stages of mussels Mytilus galloprovincialis exposed to cadmium. Ecotoxicology 26, $74-80$.

Xia, L., Chen, S., Dahms, H.U., Ying, X., Peng, X., 2016. Cadmium induced oxidative damage and apoptosis in the hepatopancreas of Meretrix meretrix. Ecotoxicology 25, 959-969.

Xu, F., Imlay, J.A., 2012. Silver(I), Mercury(II), Cadmium(II), and Zinc(II) target exposed enzymic iron-sulfur clusters when they toxify Escherichia coli. Appl. Environ. Microbiol. 78, 3614-3621.

Yang, L.L., Allen, B.C., Thomas, R.S., 2007. BMDExpress: a software tool for the benchmark dose analyses of genomic data. BMC Genom. 8, 387.

Zelko, I.N., Mariani, T.J., Folz, R.J., 2002. Superoxide dismutase multigene family: a comparison of the CuZn-SOD (SOD1), Mn-SOD (SOD2), and EC-SOD (SOD3) gene structures, evolution, and expression. Free Radical Biol. Med 33, 337-349.

Zhang, Y., Song, J., Yuan, H., Xu, Y., He, Z., 2010. Concentrations of cadmium and zinc in seawater of Bohai Bay and their effects on biomarker responses in the bivalve Chlamys farreri. Arch. Environ. Contam. Toxicol. 59, 120-128.

Zorba, M.A., Jacob, P.G., Albloushi, A., Alnafisi, R., 1992. Clams as pollution bioindicators in Kuwait's marine environment: metal accumulation and depuration. Sci. Total Environ. 120, 185-204. 